\title{
Six-mRNA risk score system and nomogram constructed for patients with ovarian cancer
}

\author{
QIANQIAN WANG, ZHUWU LU, JINQI MA, QINGSONG ZHANG, NI WANG, \\ LI QIAN, JUN ZHANG, CHEN CHEN and BEI LU
}

Department of Obstetrics and Gynecology, Wuxi People's Hospital, Wuxi, Jiangsu 214023, P.R. China

Received August 31, 2018; Accepted March 1, 2019

DOI: $10.3892 / \mathrm{ol} .2019 .10404$

\begin{abstract}
Platinum is a commonly used drug for the treatment of ovarian cancer (OC). The aim of the current study was to design and construct a risk score system for predicting the prognosis of patients with $\mathrm{OC}$ receiving platinum chemotherapy. The mRNA sequencing data and copy number variation $(\mathrm{CNV})$ information (training set) of patients with OC were downloaded from The Cancer Genome Atlas database. A validation set, GSE63885, was obtained from Gene Expression Omnibus database. The differentially expressed genes (DEGs) and CNV genes (DECNs) between platinum-resistant and platinum-sensitive groups were identified using the limma package. The intersection between DEGs and DECNs were selected. Cox regression analysis was used to identify the genes and clinical factors associated with prognosis. Risk score system assessment and nomogram analysis were performed using the survival and rms packages in R. Gene Set Enrichment Analysis was used to identify the enriched pathways in high and low risk score groups. From 1,144 DEGs and 1,864 DECNs, 48 genes that occurred in the two datasets were selected. A total of six independent prognostic genes (T-box transcription factor $\mathrm{T}$, synemin, tektin 5, growth differentiation factor 3 , solute carrier family 22 member 3 and calcium voltage-gated channel subunit $\alpha 1 \mathrm{C}$ ) and platinum response status were revealed to be associated with prognosis. Based on the six independent prognostic genes, a risk score system was constructed and assessed. Nomogram analysis revealed that the patients with the sensitive status and low risk scores had an improved prognosis. Furthermore, the current study revealed that the 574 DEGs identified were involved in eight pathways, including chemokine signaling pathway, toll-like receptor signaling pathway, cytokine-cytokine receptor interaction, RIG I like
\end{abstract}

Correspondence to: Dr Bei Lu, Department of Obstetrics and Gynecology, Wuxi People's Hospital, 299 Qingyang Road, Wuxi, Jiangsu 214023, P.R. China

E-mail: livemind@sina.com

Key words: ovarian cancer, platinum, differentially expressed genes, risk score system, nomogram receptor signaling pathway, natural killer cell mediated cytotoxicity, apoptosis, $\mathrm{T}$ cell receptor signaling pathway and Fc $\varepsilon$ receptor 1 signaling pathway. The six-mRNA risk score system designed in the present study may be used as prognosis predictor in patients with OC, whereas the nomogram may be valuable for identifying patients with OC who may benefit from platinum chemotherapy.

\section{Introduction}

Ovarian cancer (OC) affects the ovaries and may spread to the lungs, peritoneum, liver and lymph nodes $(1,2)$. Early OC may be asymptomatic, while advanced $\mathrm{OC}$ is characterized by a loss of appetite, pelvic pain, bloating and abdominal swelling (3). As OC may be difficult to diagnose in the early stages, patients with OC are often diagnosed in the advanced stages of the disease and, as a result, have an unfavorable prognosis (4). Globally, OC affects 1.2 million females and was responsible for 161,100 mortalities in 2015 (5). Among gynecological tumors, $\mathrm{OC}$ is the third most common and has the highest mortality (6). The mechanisms underlying OC remain to be elucidated.

Phospho-H2AX expression is negatively associated with the disease-free interval in epithelial OC (EOC), and its expression may allow early diagnosis of the disease as well as the identification of patients at high risk of recurrence (7). As a potential oncogene, actinin $\alpha 4$ may serve as predictive marker of poor prognosis and tumor chemoresistance in patients with advanced OC (8). Excision repair cross-complementation group 5 was revealed to be a prognostic biomarker of OC and a candidate target for the response to platinum chemotherapy treatment of patients with OC (9). Cyclin D1 $(C C N D 1)$ overexpression is significantly associated with chemosensitivity and results in poor prognosis in patients with advanced serous EOC, therefore, $C C N D 1$ may serve as a novel target for treating chemoresistant tumors $(10,11)$. Copy number variations (CNVs) of neurotrophic receptor tyrosine kinase 3 (NTRK3) were associated with platinum-resistant and platinum-sensitive relapses, and NTRK 3 amplification predicted the platinum-sensitive recurrence of OC (12). The upregulation and copy number $(\mathrm{CN})$ gain of cyclin E1 were detected in patients with ovarian clear cell carcinoma, and resulted in poor patient outcomes (13). Although the aforementioned genes may influence the prognosis of patients 
with OC, the pathways affecting OC prognosis remain to be fully elucidated.

Platinum-based drugs are effective anticancer agents and are widely used in the treatment of various solid tumors (14). Up to $80 \%$ of patients with EOC initially respond while the rest exhibit primary resistance (15). However, $\geq 80 \%$ of patients with EOC receiving platinum-based chemotherapy eventually develop resistance (15). The pathways underlying resistance to platinum drugs in OC have not been fully elucidated (16). An increased understanding of these pathways may serve to prevent or reverse resistance, improving the effect of treatment and the survival rate of patients with OC. The current study analyzed single nucleotide polymorphisms (SNPs) and CNVs in patients with OC using bioinformatics analyses. This resulted in the design of a risk score system, which was associated with platinum response status. The expression levels of several genes were associated with the survival prognosis of patients with OC. The results obtained the current study may provide novel and accurate markers for the diagnosis, prognosis and clinical treatment of patients with OC.

\section{Materials and methods}

Microarray data. The mRNA sequencing data from 419 OC tissue samples were analyzed using an Illumina HiSeq 2000 RNA Sequencing platform. SNP and CNV data from 481 OC tissue samples were analyzed using an Affymetrix Genome-Wide SNP Array 6.0 (Affymetrix; Thermo Fisher Scientific, Inc., Waltham, MA, USA). The datasets were downloaded from The Cancer Genome Atlas (TCGA; cancergenome.nih.gov) database. After the barcodes of the samples in the datasets were matched, a total of $230 \mathrm{OC}$ samples with platinum response status and survival time information were obtained. These samples included 69 platinum-resistant samples and 161 platinum-sensitive samples and were used as the training set. The GSE63885 dataset (17) was downloaded from the Gene Expression Omnibus (GEO; https://www.ncbi. nlm.nih.gov/geo) database and analyzed using a GPL570 [HG-U133_Plus_2] Affymetrix Human Genome U133 Plus 2.0 Array platform. This dataset consisted of $101 \mathrm{OC}$ tissue samples, including 75 samples with known platinum response status (34 resistant samples and 41 sensitive samples), and was used as the validation set. The clinical information of the patients from whom the samples in the training and validation sets were obtained are presented in Table I.

Data preprocessing and differential expression analysis. The preprocessCore package (18) (version 1.40.0, http://bioconductor.org/packages/release/bioc/html/preprocessCore.html) in R version 3.4.1 (https://www.r-project.org) was used to normalize the mRNA sequencing data downloaded from TCGA database. The PICNIC software (19) (the 'predict integral copy numbers in cancer' algorithm, ftp.sanger. ac.uk/pub/cancer) was used to convert the SNP data downloaded from the TCGA database from Cel format to segment format and to obtain $\mathrm{CN}$ segment data (the zero segment value indicated no $\mathrm{CNV}$, and the other non-zero signal value indicated loss or amplification mutation in the detection area). Subsequently, a human gene annotation file (GRCh38.p10) (20), was downloaded from the GENCODE database (release 27; https://www.gencodegenes.org/human) and genes were annotated according to the $\mathrm{CN}$ area coordinates of each sample. For GSE63885, format conversion, missing value supplement, background correction and data normalization were conducted using the $\mathrm{R}$ package oligo (21) (version 1.42.0; www.bioconductor.org/packages/release/bioc/html/oligo.html).

Subsequent to dividing the samples in the TCGA dataset into resistant and sensitive, the differentially expressed genes (DEGs) and differentially expressed CNV genes (DECNs) were identified using the R package limma (22) (version 3.34.7; bioconductor.org/packages/release/bioc/html/limma.html) The results with false discovery rate $(\mathrm{FDR})<0.05$ and $\log$ fold change $(\mathrm{FC}) \mid>0.263$ were selected. The overlapping genes between DEGs and DECNs were selected. The genes with CNVs [including single nucleotide polymorphism (SNP), insertion (INS) and deletion (DEL)] were chosen for further analyses.

Identification of prognosis-associated genes and clinical factors. The aforementioned selected genes and clinical factors independently associated with prognosis were analyzed using univariate and multivariate Cox regression analysis in the R package survival (23) (version 2.41.1; cran.r-project. org/web/packages/survival/index.html). $\mathrm{P}<0.05$ was considered to indicate a statistically significant difference.

Construction and assessment of the risk score system. Based on the coefficients of the independent prognostic factors in the multivariate Cox regression analysis, a gene-based risk score system was constructed for OC. The risk score of each sample was calculated according to the following formula: Risk score $=\Sigma\left(\operatorname{coef}_{\mathrm{mRNA}} \times \operatorname{Exp}_{\mathrm{mRNA}}\right)$, where $\operatorname{coef}_{\mathrm{mRNA}}$ is the prognostic coefficient of the mRNA in the multivariate Cox regression analysis and $\operatorname{Exp}_{\mathrm{mRNA}}$ is the expression level of the mRNA.

Using the median of the risk scores as a cut-off point $(24,25)$, the samples in the training set were classified into high and low risk groups. The association between the risk score and prognosis was evaluated using the Kaplan-Meier (KM) survival curve (26) in the $\mathrm{R}$ package survival, and then validated in the validation set. To reveal the association of the risk score and platinum response status, the associations between the risk score and prognosis were evaluated in the resistant and sensitive group separately using univariate and multivariate Cox regression analysis.

The association between clinical factors and prognosis was also analyzed in the high and low risk groups. The clinical factors which were significantly associated with prognosis in high and low risk groups were analyzed with KM survival analysis (26).

Association between chemotherapy response and prognosis. The clinical factors significantly associated with prognosis were identified from the samples with chemotherapy response information using the Cox regression analysis in the $\mathrm{R}$ package survival (23). A nomogram was subsequently constructed using the R package rms (27) (version 5.1-2; cran.r-project. org/web/packages/rms/index.html). The association between the platinum response status and prognosis of patients with $\mathrm{OC}$ was assessed using the probabilities acquired from the nomogram. 
Table I. Clinical information of the ovarian cancer samples in the training set and the validation set.

\begin{tabular}{|c|c|c|c|c|c|c|}
\hline \multirow[b]{2}{*}{ Variable } & \multicolumn{3}{|c|}{ Training set $(n=230)$} & \multicolumn{3}{|c|}{ Validation set $(n=75)$} \\
\hline & $\begin{array}{c}\text { Resistance } \\
\quad(n=69)\end{array}$ & $\begin{array}{l}\text { Sensitive } \\
(n=161)\end{array}$ & P-value & $\begin{array}{l}\text { Resistance } \\
\quad(n=34)\end{array}$ & $\begin{array}{l}\text { Sensitive } \\
(\mathrm{n}=41)\end{array}$ & P-value \\
\hline Age (years, mean \pm SD) & $61.77 \pm 11.47$ & $58.55 \pm 11.32$ & $5.24 \times 10^{-2}$ & - & - & - \\
\hline Neoplasm subdivision (bilateral/left/right/-) & $51 / 7 / 7 / 4$ & $109 / 27 / 14 / 11$ & $4.18 \times 10^{-1}$ & - & - & - \\
\hline Stage (II/III/IV) & $1 / 60 / 8$ & $10 / 127 / 24$ & $2.41 \times 10^{-1}$ & - & - & - \\
\hline Histological grade (G2/G3/G4/-) & $7 / 60 / 1 / 1$ & $25 / 133 / 0 / 3$ & $1.88 \times 10^{-1}$ & - & - & - \\
\hline Lymphatic invasion (yes/no/-) & $17 / 7 / 45$ & $35 / 30 / 96$ & $2.25 \times 10^{-1}$ & - & - & - \\
\hline Recurrence (yes/no) & $61 / 8$ & $122 / 5 / 34$ & $6.70 \times 10^{-2}$ & - & - & - \\
\hline Mortality (dead/alive) & $58 / 11$ & $82 / 79$ & $1.67 \times 10^{-6}$ & $33 / 1$ & $33 / 8$ & $3.50 \times 10^{-2}$ \\
\hline Overall survival time (months, mean $\pm \mathrm{SD}$ ) & $29.71 \pm 14.34$ & $48.59 \pm 25.33$ & $1.34 \times 10^{-11}$ & $27.01 \pm 14.51$ & $55.89 \pm 31.34$ & $2.13 \times 10^{-6}$ \\
\hline
\end{tabular}

'-' indicates information unavailable; G, grade; SD, standard deviation; n, number of patients.

A

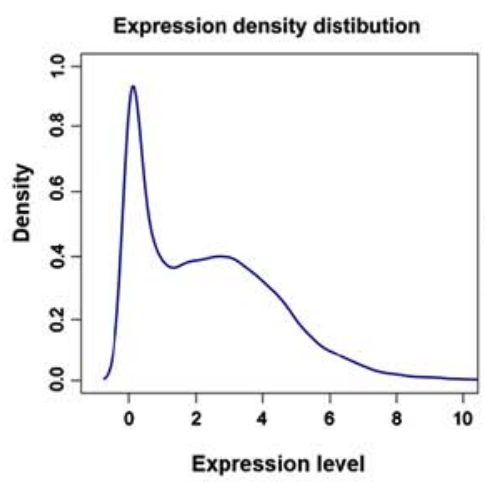

B

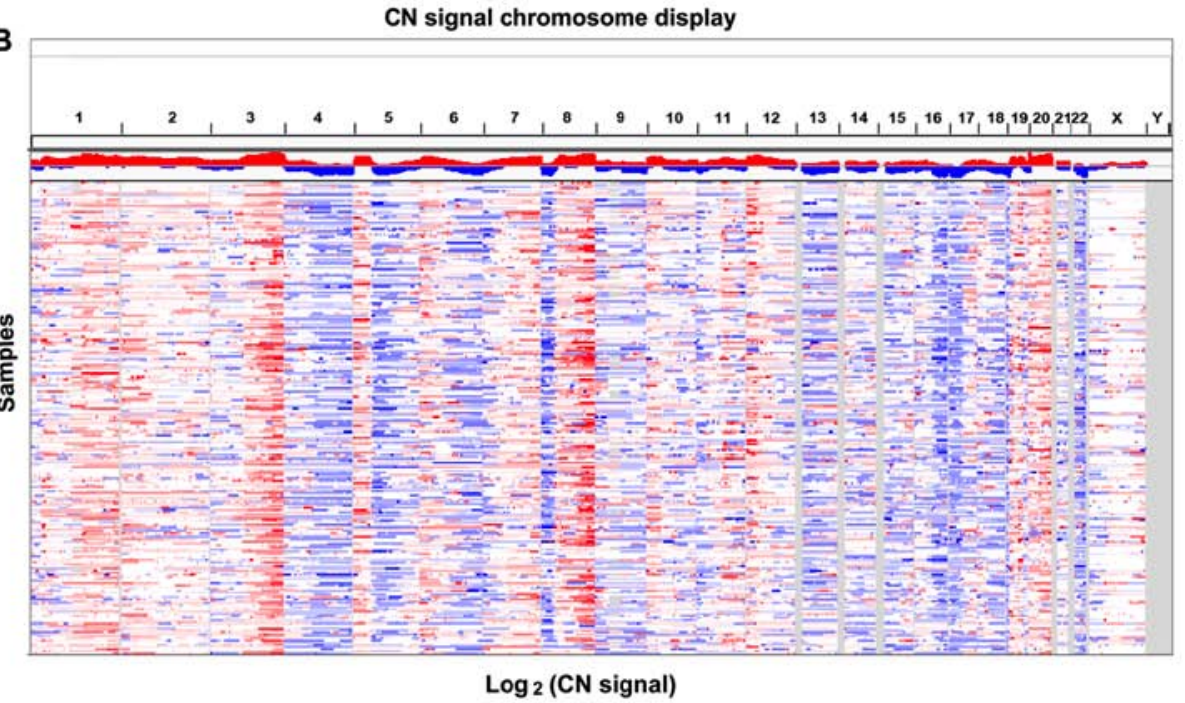

Figure 1. mRNA expression density distribution and CN signal chromosome display for The Cancer Genome Atlas dataset. (A) The mRNA expression density distribution. The horizontal and vertical axes present the expression level and density, respectively. (B) The CN signal chromosome display. The horizontal and vertical axes present the detection areas on each chromosome and the ovarian cancer samples. Red and blue indicate $\log _{2}$ (CN signal) higher and lower than 0 , respectively. $\mathrm{CN}$, copy number.

Differential expression and pathway enrichment analysis. Using the R package limma (28), the DEGs in the high and low risk groups were analyzed. The genes with FDR $<0.05$ and $\log \mathrm{FCl}>0.263$ were defined as DEGs. The correlation coefficient of DEGs and risk scores of samples were calculated using Cor function in R. Pathway enrichment analysis for the DEGs was subsequently performed using Gene Set Enrichment Analysis software 3.0 (29) (software.broadinstitute.org/gsea/index.jsp). $\mathrm{P}<0.05$ was considered to indicate a statistically significant difference.

\section{Results}

Differential expression analysis. The mRNA expression density distribution and $\mathrm{CN}$ signal chromosome display of the TCGA OC dataset are presented in Fig. 1A and B. A total of
1,144 DEGs (620 upregulated and 524 downregulated) and 1,864 DECNs (1,137 upregulated and 727 downregulated) were identified in the resistant and sensitive samples (Fig. 2A). After the DEGs and DECNs were compared, a total of 108 overlapping genes were obtained (Fig. 2B). Among the overlapping genes, 48 overlapping genes with CNVs (including 94 SNPs, 1 INS and 1 DEL) were selected and performed with the following analyses.

Identification of prognosis-associated genes and clinical factors. Combined with the expression levels and $\mathrm{CN}$ signal levels of the 48 genes in the TCGA dataset, 34 prognosis-associated genes in expression level and 29 prognosis-associated genes in CN signal level were screened using univariate multivariate Cox regression analysis. A total of 20 overlapping genes were identified after comparing the two types of 
Table II. Multivariate analysis of the six independent prognostic genes.

\begin{tabular}{lccc}
\hline Gene & Coefficient & Hazard ratio (95\% confidence interval) & P-value \\
\hline T-box transcription factor T & 1.113 & $3.046(1.677-5.531)$ & 0.0003 \\
Synemin & -0.625 & $0.535(0.367-0.779)$ & 0.0011 \\
Tektin 5 & 0.567 & $1.763(1.174-2.6452)$ & 0.0062 \\
Growth differentiation factor 3 & 0.374 & $1.453(1.0701-1.973)$ & 0.0166 \\
Solute carrier family 22 member 3 & 0.652 & $1.919(1.036-3.555)$ & 0.0384 \\
Calcium voltage-gated channel subunit $\alpha 1 \mathrm{C}$ & 0.56 & $1.752(1.022-3.002)$ & 0.0415 \\
\hline
\end{tabular}
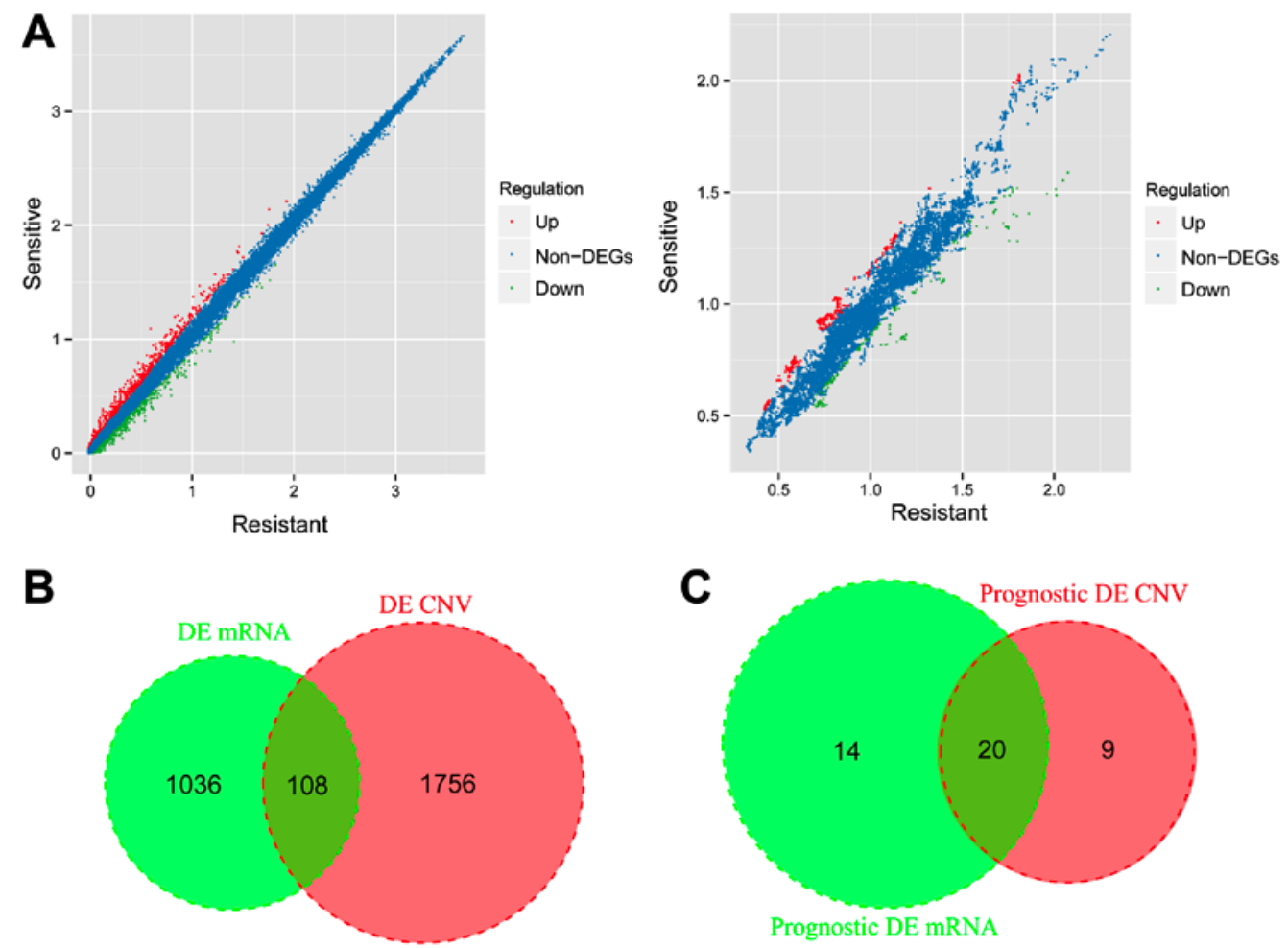

Figure 2. Scatter and Venn diagrams. (A) The scatter diagrams for the mRNA-sequencing data (left) and the single nucleotide polymorphisms and CNVs (right). Red presents the genes with upregulated expression or copy number signal values in the sensitive sample. (B) The Venn diagram presents the DEGs and DECNs and the overlapping genes. (C) The Venn diagram for comparing the prognosis-associated DEGs and DECNs. CNVs, copy number variations; DEGs, differentially expressed genes; DECNs, differentially expressed CNV genes.

genes which were significantly associated with prognosis in expression level and $\mathrm{CN}$ signal level (Fig. 2C), including calcium voltage-gated channel subunit $\alpha 1 \mathrm{C}(C A C N A 1 C)$, CD274 molecule, CECR2 histone acetyl-lysine reader, contactin 6, DEAQ-box RNA dependent ATPase 1, FERM domain-containing 1, growth differentiation factor 3 (GDF3), gap junction protein $\alpha 8$, neuroligin 1,NTRK3, PARN-like ribonuclease domain-containing 1, seizure-related 6 homolog-like, solute carrier family 22 member 3 (SLC22A3), solute carrier family 5 member 1 , synemin $(S Y N M)$, synaptotagmin 9, T-box transcription factor T (TBXT), tektin 5 (TEKT5), V-set and transmembrane domain containing 2-like and ZFP57 zinc finger protein.

The 20 overlapping genes were subjected to multivariate Cox regression analysis, and a total of six independent prognostic genes (TBXT, SYNM, TEKT5, GDF3, SLC22A3 and $C A C N A 1 C)$ were identified $(\mathrm{P}<0.05$; Table II). The CNV data of the six independent prognostic genes are presented in Table III.

Construction and assessment of the risk score system. Based on the multivariate Cox regression coefficients of the six independent prognostic genes, the following risk score system was developed $(24,25)$ : Risk score $=(1.113) \mathrm{x}$ $\operatorname{Exp}_{\text {Tвхт }}+(-0.625) \operatorname{xxp}_{\text {SYNM }}+(0.567)$ Exp $_{\text {TEKT } 5}+(0.374) \mathrm{x}$ $\operatorname{Exp}_{\mathrm{GDF} 3}+(0.652) \times \operatorname{Exp}_{\mathrm{SLC22A} 3}+(0.560) \times \operatorname{Exp}_{\mathrm{CACNA} 1 \mathrm{C}}$, where Exp is the relative expression level of the genes. The risk scores of TCGA samples were calculated using the above formula. The median was used as the cut-off point to divide the samples in the training set into high and low risk groups. The association between the risk groups and prognosis was evaluated using the KM survival curve (Fig. 3A), and subsequently validated in the validation set (Fig. 3B). The association between the predictive results of the risk score 


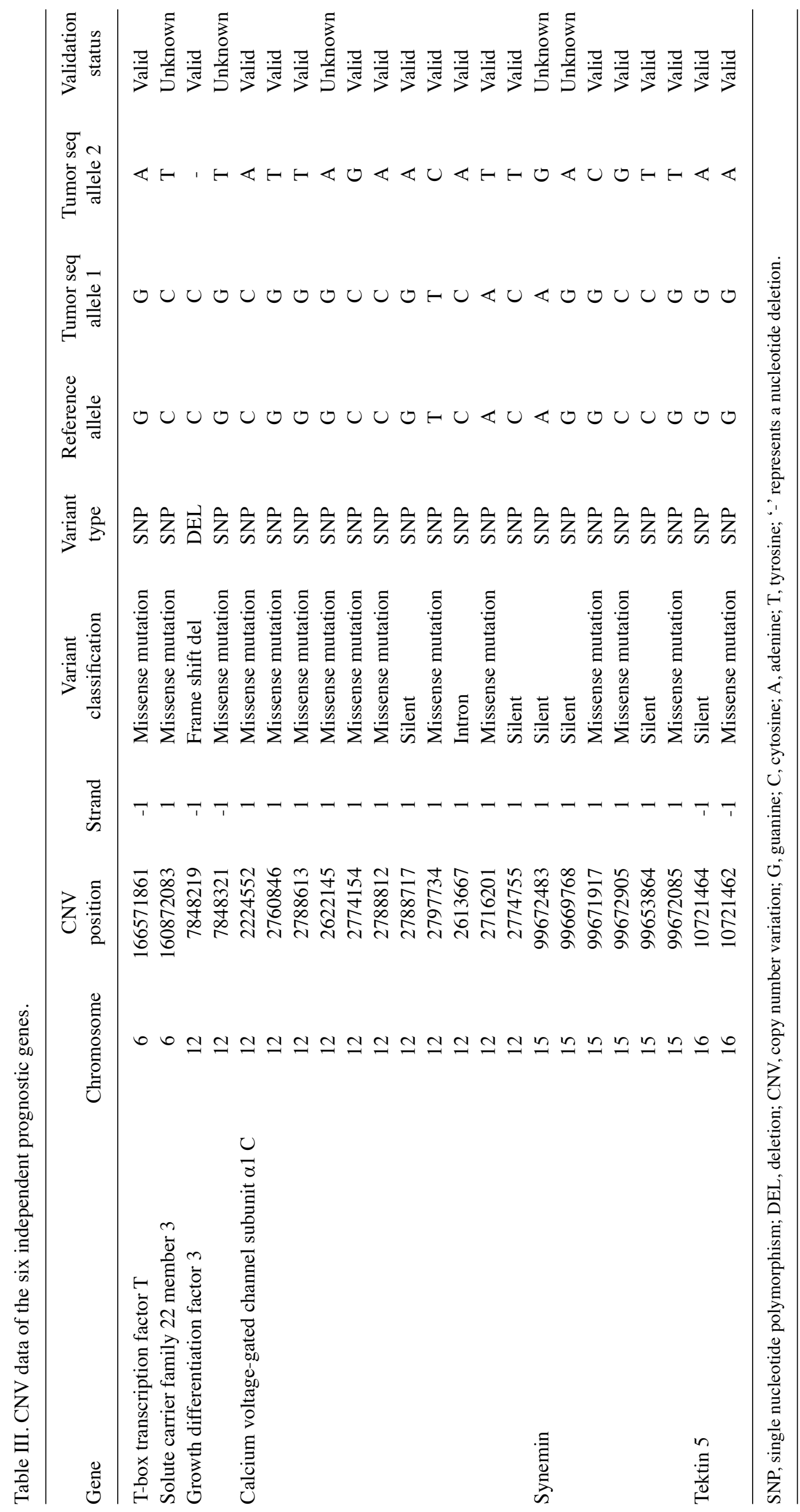


A

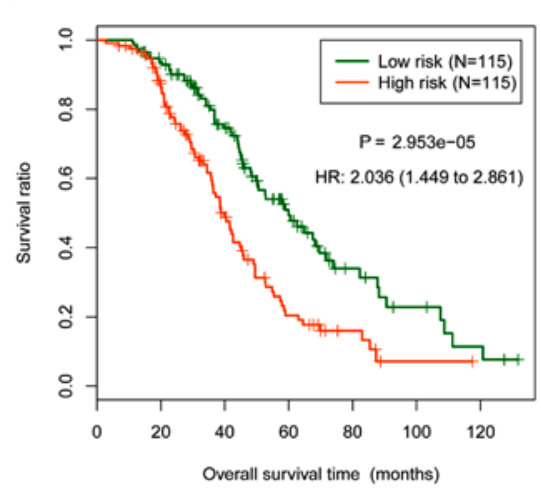

B

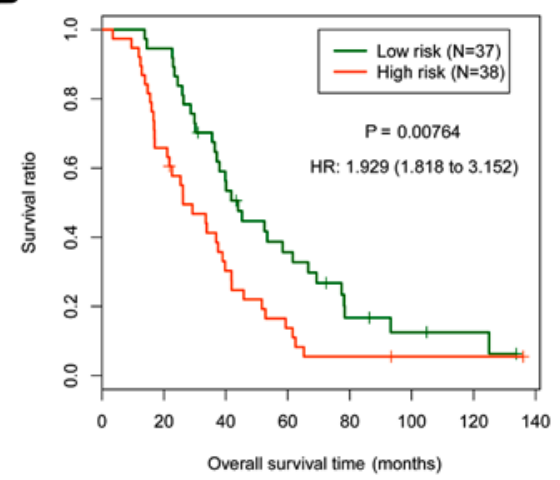

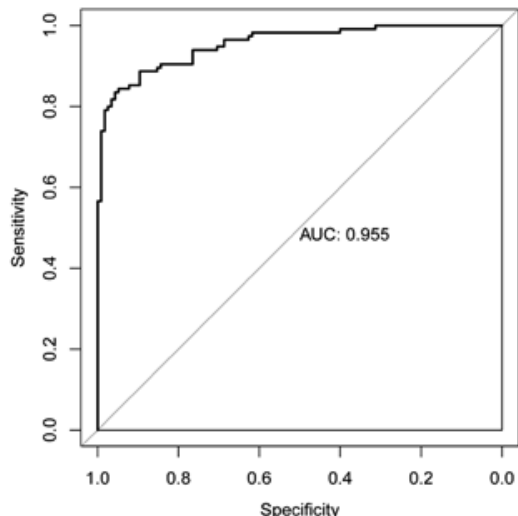

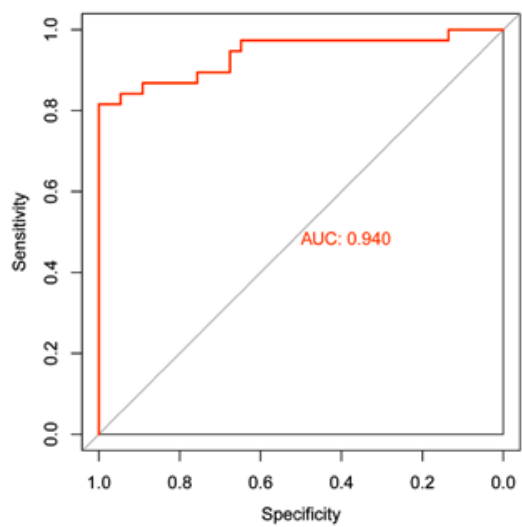

Figure 3. KM survival curves and the AUC for the training and validation sets. (A) The KM survival curve (left) and AUC (right) for the training set. (B) The KM survival curve (left) and AUC (right) for the validation set. Red and green in KM survival curves present high and low risk groups, respectively. HR, hazard ratio; KM, Kaplan-Meier; AUC, area under the curve.

A

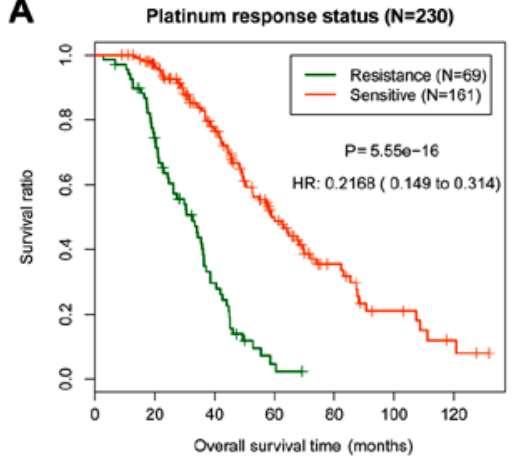

C

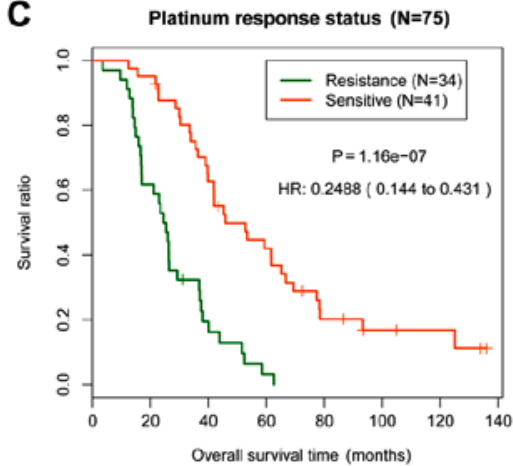

B

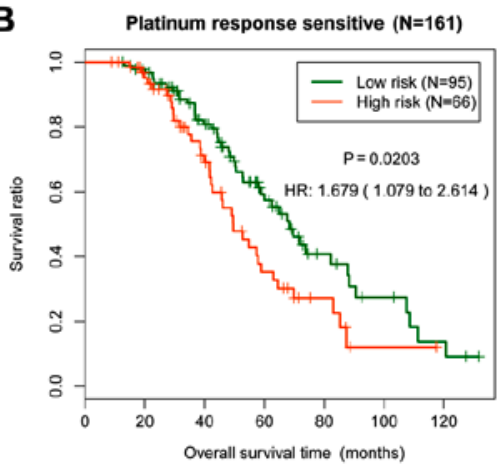

D

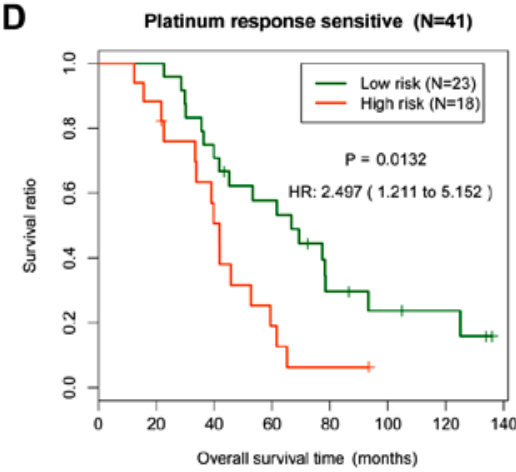

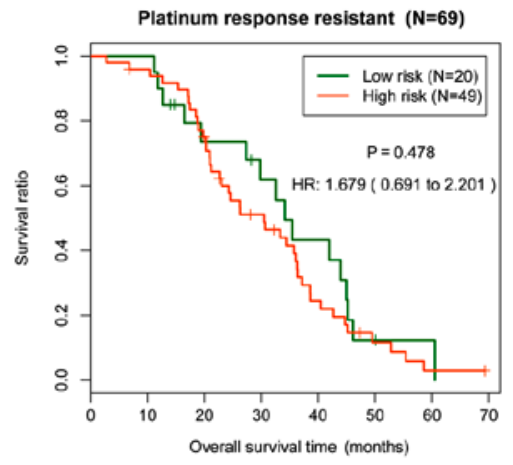

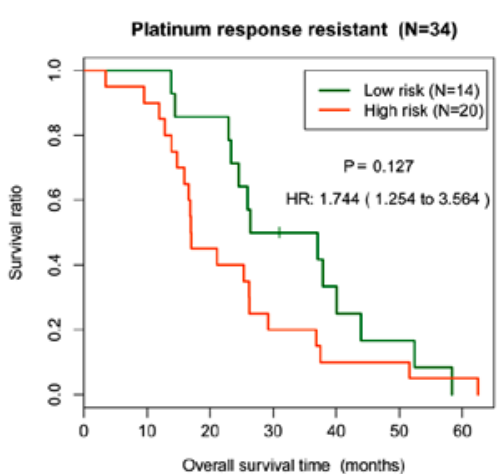

Figure 4. KM survival curves for the training and validation sets. (A) The KM survival curve (left) for the entire training set. Red and green present sensitive and resistant samples, respectively. (B) The KM survival curves for the sensitive (left) and resistant (right) samples in the training set. Red and green present high and low risk groups, respectively. (C) The KM survival curve (left) for the entire validation set. Red and green present sensitive and resistant samples, respectively. (D) The KM survival curves for the sensitive (left) and resistant (right) samples in the validation set. Red and green present high and low risk groups, respectively. HR, hazard ratio; KM, Kaplan-Meier. 
Table IV. Results of the Cox regression analysis for identification of the clinical factors significantly associated with prognosis.

\begin{tabular}{|c|c|c|c|c|c|c|}
\hline \multirow[b]{2}{*}{ Variable } & \multicolumn{3}{|c|}{ Univariate analysis } & \multicolumn{3}{|c|}{ Multivariate analysis } \\
\hline & HR & $95 \% \mathrm{CI}$ & P-value & $\mathrm{HR}$ & $95 \% \mathrm{CI}$ & P-value \\
\hline Age & 1.008 & $0.993-1.024$ & 0.2970 & - & - & - \\
\hline Platinum response status & 0.217 & $0.149-0.314$ & $5.55 \times 10^{-16}$ & 0.246 & $0.168-0.362$ & $1.05 \times 10^{-12}$ \\
\hline Neoplasm subdivision & 0.844 & $0.632-1.127$ & 0.2490 & - & - & - \\
\hline Stage & 1.052 & $0.727-1.524$ & 0.7870 & - & - & - \\
\hline Lymphatic invasion & 0.959 & $0.529-1.736$ & 0.8890 & - & - & - \\
\hline Histological grade & 1.272 & $0.805-2.009$ & 0.3020 & - & - & - \\
\hline Recurrence & 0.695 & $0.352-1.372$ & 0.2910 & - & - & - \\
\hline Predicted status & 2.036 & $1.449-2.861$ & $2.95 \times 10^{-05}$ & 1.489 & $1.039-2.132$ & 0.0298 \\
\hline
\end{tabular}

HR, hazard ratio; CI, confidence interval; '-' indicates that these parameters were not included in the multivariate analysis.

A

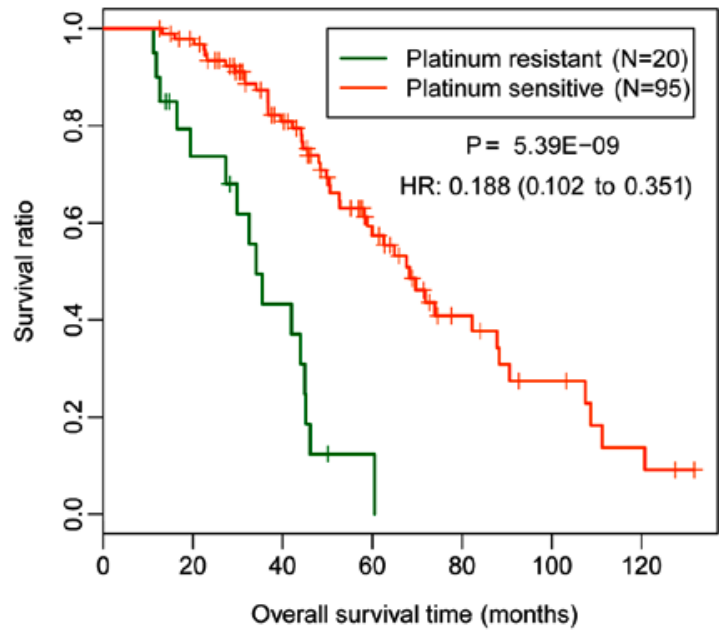

B

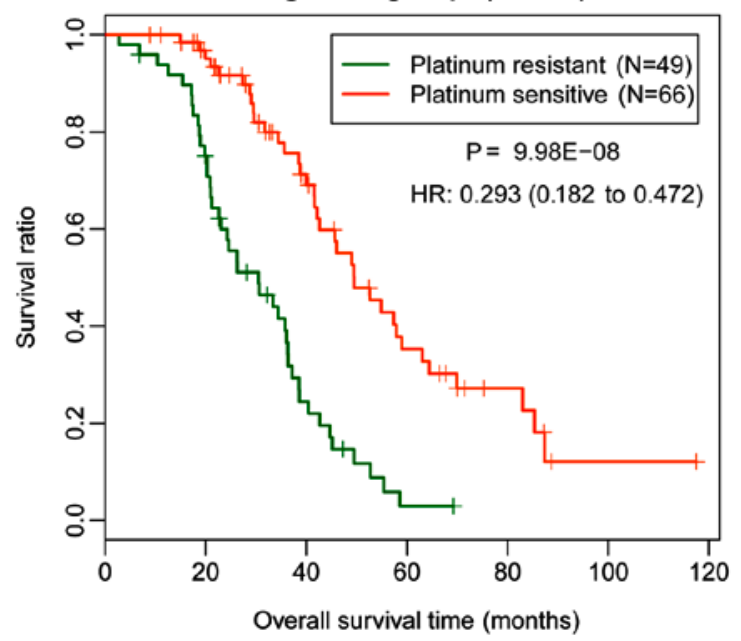

Figure 5. KM survival curves for the training and validation sets. (A) The KM survival curve demonstrating the association between the platinum response status of the samples in low risk group and actual prognosis. (B) The KM survival curve demonstrating the association between the platinum response status of the samples in high risk group and actual prognosis. Red and green present sensitive and resistant samples, respectively. HR, hazard ratio; KM, Kaplan-Meier.

system and platinum response status was further analyzed (Fig. 4). The platinum response status of the samples in the training set $\left(\mathrm{P}=5.55 \times 10^{-16}\right.$; Fig. $\left.4 \mathrm{~A}\right)$ and the validation set $\left(\mathrm{P}=1.16 \times 10^{-07}\right.$; Fig. $\left.4 \mathrm{C}\right)$ were significantly associated with the actual prognosis. In the platinum sensitive samples, the predictive results of the training set $(\mathrm{P}=0.0203$; Fig. 4B) and the validation set $(\mathrm{P}=0.0132$; Fig. $4 \mathrm{D})$ were significantly associated with survival prognosis. In the platinum resistant samples, the association between the predictive results and prognosis was not significant (Fig. 4B and D). Therefore, the risk score system could accurately predict the prognosis of the sensitive samples.

The samples in the training dataset were divided into high and low risk groups according to the median of the risk scores. The association between clinical factors and prognosis was determined. The platinum response status and predicted status based on the risk scores had a significant association with prognosis [platinum response status, $\mathrm{P}=1.05 \times 10^{-12}$; hazard ratio $(\mathrm{HR})=0.246$; predicted status, $\mathrm{P}=0.0298 ; \mathrm{HR}=1.489$ ], which suggested that response to platinum treatment improves prognosis and patients with platinum sensitivity may have an improved prognosis compared with platinum-resistant patients (Table IV). Statistical analysis revealed that the platinum response status was significantly associated with the prognosis of low $\left(\mathrm{P}=5.39 \times 10^{-09}\right.$; Fig. 5A) and high $\left(\mathrm{P}=9.98 \times 10^{-08}\right.$; Fig. 5B) risk groups (Table $\mathrm{V})$. The results obtained in the current study indicated that patients with platinum sensitivity in the low and high risk groups had improved survival prognosis compared with patients with platinum resistance.

Association between chemotherapy response and prognosis. Cox regression analysis was performed for the platinum response status and risk scores, revealing that the sensitive status improved prognosis in $\mathrm{OC}(\mathrm{HR}=0.217)$ and that the risk score worsened the prognosis (the higher the risk score, the worse the prognosis; Fig. 6A). Subsequently, nomogram analysis was performed based on the platinum response status and risk score. The platinum response status 
Table V. Results of stratified analysis of clinical factors in high and low risk groups.

\begin{tabular}{|c|c|c|c|c|}
\hline Variable & $\begin{array}{l}\text { Low risk }(\mathrm{n}=115) \\
\operatorname{HR}(95 \% \mathrm{CI})\end{array}$ & P-value & $\begin{array}{l}\text { High risk }(\mathrm{n}=115) \\
\operatorname{HR}(95 \% \mathrm{CI})\end{array}$ & P-value \\
\hline Age & $0.996(0.974-1.02)$ & 0.7900 & $1.016(0.996-1.037)$ & 0.1180 \\
\hline Platinum response status & $0.188(0.102-0.351)$ & $5.39 \times 10^{-09}$ & $0.293(0.182-0.472)$ & $9.98 \times 10^{-08}$ \\
\hline Neoplasm subdivision & $0.692(0.446-1.075)$ & 0.0984 & $1.227(0.832-1.811)$ & 0.3001 \\
\hline Pathological Stage & $0.953(0.562-1.615)$ & 0.8590 & $1.174(0.668-2.062)$ & 0.5770 \\
\hline Histological grade & $1.569(0.670-3.671)$ & 0.2950 & $1.312(0.743-2.317)$ & 0.3480 \\
\hline Recurrence & $1.412(1.195-2.25)$ & 0.7310 & $0.757(0.362-1.585)$ & 0.4590 \\
\hline
\end{tabular}

HR, hazard ratio; CI, confidence interval; n, number of patients.

A

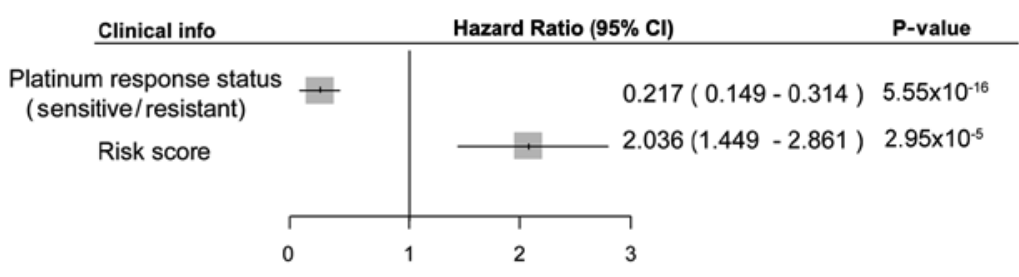

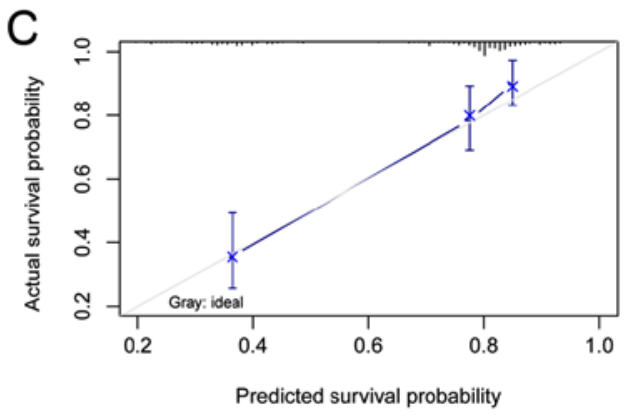

B

Points (months)

Platinum (resistant/sensitive)

Risk score

Total points (months)

3-year survival probability

5-year survival probability
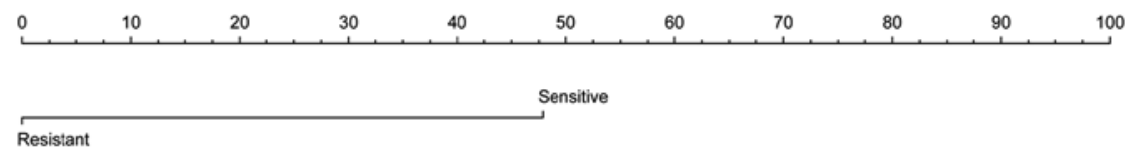

\begin{tabular}{llllllllll}
\hline 3 & 2.5 & 2 & 1.5 & 1 & 0.5 & 0 & -0.5 & -1 & -1.5
\end{tabular}

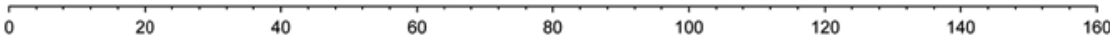

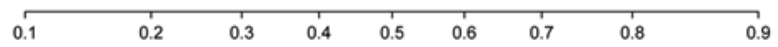

\begin{tabular}{llllllll}
\hline 0.1 & 1 & 1 & 1 & 1 & 1 & & 1 \\
& 0.2 & 0.3 & 0.4 & 0.5 & 0.6 & 0.7 & 0.8
\end{tabular}

Figure 6. Results of the Cox regression analysis. (A) The result of multivariate Cox regression analysis for platinum response status and risk score. (B) The nomogram predicts the 3-year survival probability and 5-year survival probability based on platinum response status and risk score. (C) The calibration diagram between the predicted 5-year survival probability and the actual survival probability. The blue line represents predicted survival probability; the gray line represents the actual survival probability. HR, hazard ratio; CI, confidence interval; ideal, the actual survival rate according to the dataset.

and risk score were used as independent prognostic factors to predict the 3-year survival probability and 5-year survival probability, and the results demonstrated that patients with the sensitive status and low risk scores had improved prognosis compared with patients with platinum resistance and high risk scores (Fig. 6B). The calibration curve for the predicted 5-year survival probability and the actual survival probability revealed that the predicted curve and the ideal curve were similar. The predicted results were therefore consistent with the actual survival status according to the clinical information in the dataset (Fig. 6C).

Differential expression and pathway enrichment analyses. The OC samples in the TCGA set were divided into high and low risk groups. A total of 574 DEGs (297 upregulated and 277 downregulated) were identified in the high and low risk groups. The volcano plot of the 574 DEGs is presented in Fig. 7A. The correlation coefficient of DEGs and risk scores of samples were calculated using Cor function in R. The top 100 DEGs (the top 50 for positive and negative association, respectively) were selected for analysis based on the association of the DEGs with the risk scores. The expression heatmap is presented in Fig. 7B. Pathway enrichment analysis revealed that DEGs were significantly enriched in eight pathways, including chemokine signaling pathway, toll-like receptor signaling pathway, cytokine-cytokine receptor interaction, RIG I-like receptor signaling pathway, natural killer cell-mediated cytotoxicity, apoptosis, $\mathrm{T}$ cell receptor signaling pathway and Fc $\varepsilon$ receptor 1 signaling pathway (Table VI). 
Table VI. Eight significantly enriched pathways between high and low risk groups.

\begin{tabular}{lccc}
\hline Name & ES & NES & NOM P-value \\
\hline Chemokine signaling pathway & -0.71585 & -2.25369 & $<0.001$ \\
Toll-like receptor signaling pathway & -0.76181 & -2.08654 & $<0.001$ \\
Cytokine-cytokine receptor interaction & -0.51545 & -1.92261 & 0.0061 \\
RIG I-like receptor signaling pathway & -0.77359 & -1.89329 & 0.0085 \\
Natural killer cell mediated cytotoxicity & -0.59467 & -1.78868 & 0.0143 \\
Apoptosis & -0.63801 & -1.69221 & 0.0178 \\
T cell receptor signaling pathway & -0.72389 & -1.65438 & 0.0242 \\
Fc $\varepsilon$ receptor 1 signaling pathway & -0.68246 & -1.54939 & 0.0391 \\
\hline
\end{tabular}

ES, enrichment score; NES, normalized enrichment score; NOM, nominal.

A

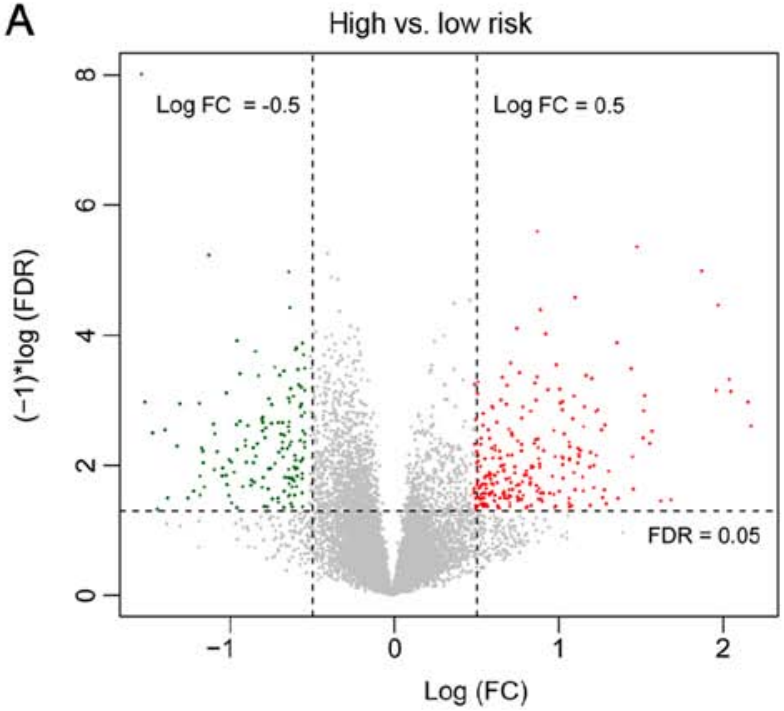

B

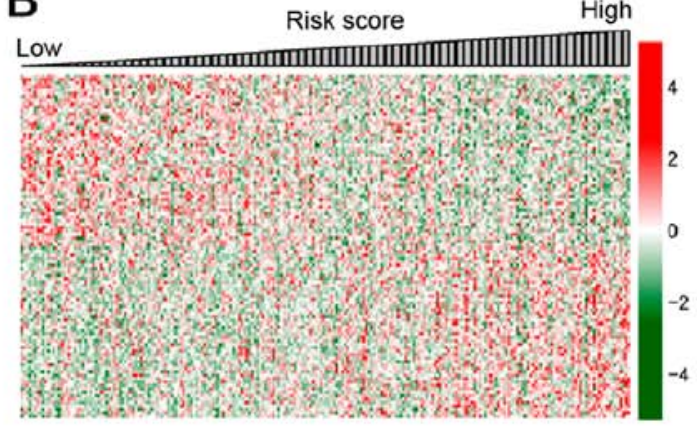

Figure 7. Differential expression analysis and association heatmap. (A) The volcano plot of the DEGs between high and low risk groups. Red, green and grey dots represent upregulated genes, downregulated genes and non-DEGs, respectively. (B) The expression heatmap of the top 100 DEGs. FDR, false discovery rate; FC, fold change; DEGs, differentially expressed genes; IL6R, interleukin 6 receptor; IL2RB, interleukin 2 receptor subunit beta; IL2RG interleukin 2 receptor subunit gamma; CXCL11, C-X-C motif chemokine ligand 11.

\section{Discussion}

In the present study, 1,144 DEGs and 1,864 DECNs in platinum resistant samples and platinum sensitive samples were screened. A total of 108 overlapping genes between the DEGs and DECNs were identified, from which 48 genes with CNVs were selected for subsequent analyses. There were 20 genes significantly associated with prognosis in the expression level and $\mathrm{CN}$ signal level, from which six independent prognostic genes (T, SYNM, TEKT5, GDF3, SLC22A3 and CACNAIC) were selected. The platinum response status had a significant association with prognosis and platinum sensitivity resulted in improved prognosis. Subsequently, a risk score system based on the six independent prognostic factors was constructed and evaluated. Nomogram analysis indicated that patients with sensitive status and low risk scores had improved prognosis compared with patients with a resistant status and high risk scores. A total of 574 DEGs were identified in high and low risk groups, and these genes were enriched in eight pathways.
The Tektin (TEKT) family consists of several filament-forming proteins in male germ cells, including TEKT1, TEKT2, TEKT3, TEKT4 and TEKT5 (30). TEKT5 was reported to be a cancer/testis antigen, suggesting that TEKT5 may be used for the diagnosis and immunotherapy of patients with testicular cancer (31). SYNM is a potential tumor suppressor gene in breast cancer, and its promoter methylation status may be used to predict the risk of recurrence in patients with breast cancer (32). SYNM is an intermediate filament-associated protein that may function in the formation of hepatocellular carcinoma, and its alteration may result in the pleomorphism of tumor cells (33). Since TEKT5 and SYNM were revealed to be involved in the pathogenesis of different types of cancer in the aforementioned studies, they may also be implicated in the pathogenesis of OC.

The plasma concentration of GDF15 is increased in OC, and increased plasma concentration is negatively associated with survival time and is an independent prognostic predictor $(34,35)$. GDF 15 upregulation contributes to the 
growth and invasion of rapamycin-sensitive OC cells; therefore, rapamycin inhibition may be effective for treating patients with GDF15-overexpressing OC (36). GDF3 is a member of the transforming growth factor $\beta$ family and is expressed in several types of tumors (37). GDF3 blocking combined with retinoic acid may be a promising therapeutic strategy for different types of solid cancer (37). GDF3 is markedly downregulated in breast cancer tissues, and its reconstitution may be a applied for inhibiting the aggressive growth of the tumor (38). Therefore, upregulation of GDF3 may also be associated with the poor prognosis of patients with OC.

$S L C 22 A 16$ is expressed in ovarian clear-cell adenocarcinoma and it can promote the uptake of doxorubicin compounds directed against the cancer cells $(39,40)$. SLC22A3 affects the absorption of numerous basic drugs and endogenous amines in multiple tissues (41). SLC22A3 is downregulated in aggressive prostate cancer and is a key risk factor for the disease (41). $S L C 22 A 3$ is essential for the cytotoxicity induced by oxaliplatin as it facilitates its uptake into tumors (42). SLC22A3 expression may therefore serve as a marker for the efficacy of chemotherapy in different types of cancer (42). The results obtained in the aforementioned studies suggest that $S L C 22 \mathrm{~A} 3$ may affect the efficacy of chemotherapy in patients with OC.

A limitation of the current study was a lack of experimental validation $(43,44)$. Although bioinformatics reveals genes and pathways involved in platinum-based chemotherapy for ovarian cancer, future experiments are required to verify these results.

In conclusion, a six-mRNA risk score system was constructed to predict the prognosis of patients with OC. The risk score system-based nomogram may be applied for identifying patients with $\mathrm{OC}$ who will benefit from platinum chemotherapy.

\section{Acknowledgements}

Not applicable.

\section{Funding}

No funding was received.

\section{Availability of data and materials}

The datasets used during the current study are available from the corresponding author on reasonable request.

\section{Authors' contributions}

QW performed data analysis and wrote the manuscript. ZL, JM, QZ, NW, LQ, JZ and CC substantially contributed to data analysis and manuscript revision. BL conceived and designed the study. All authors read and approved the final manuscript.

\section{Ethics approval and consent to participate}

Not applicable.

\section{Patient consent for publication}

Not applicable.

\section{Competing interests}

The authors declare that they have no competing interests.

\section{References}

1. Jayson GC, Kohn EC, Kitchener HC and Ledermann JA: Ovarian cancer. Lancet 384: 1376-1388, 2014.

2. Cree IA: Cancer biology. Methods Mol Biol 731: 1-11, 2011.

3. Ebell MH, Culp MB and Radke TJ: A systematic review of symptoms for the diagnosis of ovarian cancer. Am J Prev Med 50: 384-394, 2016.

4. Mozzetti S, Ferlini C, Concolino P, Filippetti F, Raspaglio G, Prislei S, Gallo D, Martinelli E, Ranelletti FO, Ferrandina G and Scambia G: Class III $\beta$-Tubulin overexpression is a prominent mechanism of paclitaxel resistance in ovarian cancer patients. Clin Cancer Res 11: 298-305, 2005.

5. GBD 2015 Mortality and Causes of Death Collaborators: Global, regional, and national life expectancy, all-cause mortality, and cause-specific mortality for 249 causes of death, 1980-2015: A systematic analysis for the global burden of disease study 2015 . Lancet 388: 1459-1544, 2016.

6. McGuire S: World Cancer Report 2014. Geneva, Switzerland: World Health Organization, International Agency for Research on Cancer, WHO Press, 2015. Adv Nutr 7: 418-419, 2016.

7. Mei L, Hu Q, Peng J, Ruan J, Zou J, Huang Q, Liu S and Wang H: Phospho-histone $\mathrm{H} 2 \mathrm{AX}$ is a diagnostic and prognostic marker for epithelial ovarian cancer. Int J Clin Exp Pathol 8: 5597-5602, 2015.

8. Yamamoto S, Tsuda H, Honda K, Onozato K, Takano M, Tamai S, Imoto I, Inazawa J, Yamada T and Matsubara O: Actinin-4 gene amplification in ovarian cancer: A candidate oncogene associated with poor patient prognosis and tumor chemoresistance. Mod Pathol 22: 499-507, 2009.

9. Walsh CS, Ogawa S, Karahashi H, Scoles DR, Pavelka JC, Tran H, Miller CW, Kawamata N, Ginther C, Dering J, et al: ERCC5 is a novel biomarker of ovarian cancer prognosis. J Clin Oncol 26: 2952-2958, 2008

10. Hashimoto T, Yanaihara N, Okamoto A, Nikaido T, Saito M, Takakura S, Yasuda M, Sasaki H, Ochiai K and Tanaka T: Cyclin D1 predicts the prognosis of advanced serous ovarian cancer. Exp Ther Med 2: 213-219, 2011.

11. Dai J, Wei RJ, Li R, Feng JB, Yu YL and Liu PS: A study of CCND1 with epithelial ovarian cancer cell proliferation and apoptosis. Eur Rev Med Pharmacol Sci 20: 4230-4235, 2016.

12. Ge L, Li N, Liu M, Xu NZ, Wang MR and Wu LY: Copy number variations of neurotrophic tyrosine receptor kinase 3 (NTRK3) may predict prognosis of ovarian cancer. Medicine (Baltimore) 96: e7621, 2017.

13. Ayhan A, Kuhn E, Wu RC, Ogawa H, Bahadirli-Talbott A, Mao TL, Sugimura H, Shih IM and Wang TL: CCNE1 copy-number gain and overexpression identify ovarian clear cell carcinoma with a poor prognosis. Mod Pathol 30: 297-303, 2017.

14. Gonzalez VM, Fuertes MA, Alonso C and Perez JM: Is cisplatin-induced cell death always produced by apoptosis? Mol Pharmacol 59: 657-663, 2001.

15. Yakirevich E, Sabo E, Naroditsky I, Sova Y, Lavie O and Resnick MB: Multidrug resistance-related phenotype and apoptosis-related protein expression in ovarian serous carcinomas. Gynecol Oncol 100: 152-159, 2006.

16. Norouzi-Barough L,Sarookhani MR, Sharifi M, Moghbelinejad S, Jangjoo S and Salehi R: Molecular mechanisms of drug resistance in ovarian cancer. J Cell Physiol 233: 4546-4562, 2018.

17. Lisowska KM, Olbryt M, Dudaladava V, Pamuła-Piłat J, Kujawa K, Grzybowska E, Jarząb M, Student S, Rzepecka IK, Jarząb B and Kupryjańczyk J: Gene expression analysis in ovarian cancer-faults and hints from DNA microarray study. Front Oncol 4: 6, 2014.

18. Bolstad BM, Irizarry RA, Astrand M and Speed TP: A comparison of normalization methods for high density oligonucleotide array data based on variance and bias. Bioinformatics 19: 185-193, 2003.

19. Greenman CD, Bignell G, Butler A, Edkins S, Hinton J, Beare D, Swamy S, Santarius T, Chen L, Widaa S, et al: PICNIC: An algorithm to predict absolute allelic copy number variation with microarray cancer data. Biostatistics 11: 164-175, 2010. 
20. Harrow J, Frankish A, Gonzalez JM, Tapanari E, Diekhans M, Kokocinski F, Aken BL, Barrell D, Zadissa A, Searle S, et al: GENCODE: The reference human genome annotation for The ENCODE Project. Genome Res 22: 1760-1774, 2012.

21. Parrish RS and Spencer HJ III: Effect of normalization on significance testing for oligonucleotide microarrays. J Biopharm Stat 14: 575-589, 2004.

22. Ritchie ME, Phipson B, Wu D, Hu Y, Law CW, Shi W and Smyth GK: Limma powers differential expression analyses for RNA-sequencing and microarray studies. Nucleic Acids Res 43 : e47, 2015 .

23. Wang P, Wang Y, Hang B, Zou X and Mao JH: A novel gene expression-based prognostic scoring system to predict survival in gastric cancer. Oncotarget 7: 55343-55351, 2016.

24. Bao Z, Zhang W and Dong D: A potential prognostic lncRNA signature for predicting survival in patients with bladder urothelial carcinoma. Oncotarget 8: 10485-10497, 2017.

25. Zeng JH, Liang L, He RQ, Tang RX, Cai XY, Chen JQ, Luo DZ and Chen G: Comprehensive investigation of a novel differentially expressed lncRNA expression profile signature to assess the survival of patients with colorectal adenocarcinoma. Oncotarget 8: 16811-16828, 2017.

26. Jager KJ, van Dijk PC, Zoccali C and Dekker FW: The analysis of survival data: The Kaplan-Meier method. Kidney Int 74: $560-565,2008$.

27. Eng KH, Schiller E and Morrell K: On representing the prognostic value of continuous gene expression biomarkers with the restricted mean survival curve. Oncotarget 6: 36308-36318, 2015.

28. Smyth GK: Limma: Linear models for microarray data. In: Bioinformatics and Computational Biology Solutions Using $\mathrm{R}$ and Bioconductor. Gentleman R, Carey V, Dudoit S, Irizarry R and Huber W (eds). Springer, New York, NY, pp397-420, 2005.

29. Ackermann M and Strimmer K: A general modular framework for gene set enrichment analysis. BMC Bioinformatics 10: 47, 2009.

30. Amos LA: The tektin family of microtubule-stabilizing proteins. Genome Biol 9: 229, 2008.

31. Hanafusa T, Mohamed AE, Domae S, Nakayama E and Ono T: Serological identification of Tektin5 as a cancer/testis antigen and its immunogenicity. BMC Cancer 12: 520, 2012.

32. Noetzel E, Rose M, Sevinc E, Hilgers RD, Hartmann A, Naami A, Knüchel R and Dahl E: Intermediate filament dynamics and breast cancer: Aberrant promoter methylation of the Synemin gene is associated with early tumor relapse. Oncogene 29: 4814-4825, 2010.

33. Ho CC, Ho HC, Liu YH, Pei RJ, Cheng CC, Lee KY, Yeh KT and Lai YS: Altered synemin could affect the organization of intermediate filament in human hepatocellular carcinoma. J Med 35: 171-180, 2004.

34. Staff AC, Bock AJ, Becker C, Kempf T, Wollert KC and Davidson B: Growth differentiation factor-15 as a prognostic biomarker in ovarian cancer. Gynecol Oncol 118: 237-243, 2010.
35. Bock AJ, Stavnes HT, Kempf T, Tropè CG, Berner A, Davidson B and Staff AC: Expression and clinical role of growth differentiation factor-15 in ovarian carcinoma effusions. Int J Gynecol Cancer 20: 1448-1455, 2010.

36. Griner SE, Joshi JP and Nahta R: Growth differentiation factor 15 stimulates rapamycin-sensitive ovarian cancer cell growth and invasion. Biochem Pharmacol 85: 46-58, 2013.

37. Tykwinska K, Lauster R, Knaus P and Rosowski M: Growth and differentiation factor 3 induces expression of genes related to differentiation in a model of cancer stem cells and protects them from retinoic acid-induced apoptosis. PLoS One 8: e70612, 2013.

38. Li Q, Ling Y and Yu L: GDF3 inhibits the growth of breast cancer cells and promotes the apoptosis induced by Taxol. J Cancer Res Clin Oncol 138: 1073-1079, 2012.

39. Ota K, Ito K, Akahira J, Sato N, Onogawa T, Moriya T, Unno M, Abe T, Niikura H, Takano T and Yaegashi N: Expression of organic cation transporter SLC22A16 in human epithelial ovarian cancer: A possible role of the adriamycin importer. Int J Gynecol Pathol 26: 334-340, 2007.

40. Ota K, Akahira JI, Sato N, Moriya T, Unno M, Abe T, Ito K and Yaegashi N: Expression of the organic cation transporter SLC22A16 can be a feature of human epithelial ovarian cancers with different histological type. J Clin Oncol 23: 5131, 2005.

41. Chen L, Hong C, Chen EC, Yee SW, Xu L, Almof EU, Wen C, Fujii K, Johns SJ, Stryke D, et al: Genetic and epigenetic regulation of the organic cation transporter 3, SLC22A3. Pharmacogenomics J 13: 110-120, 2013.

42. Yokoo S, Masuda S, Yonezawa A, Terada T, Katsura T and Inui K: Significance of organic cation transporter 3 (SLC22A3) expression for the cytotoxic effect of oxaliplatin in colorectal cancer. Drug Metab Dispos 36: 2299-2306, 2008.

43. Tan DS, Rothermundt C, Thomas K, Bancroft E, Eeles R, Shanley S, Ardern-Jones A, Norman A, Kaye SB and Gore ME: 'BRCAness' syndrome in ovarian cancer: A case-control study describing the clinical features and outcome of patients with epithelial ovarian cancer associated with BRCA1 and BRCA2 mutations. J Clin Oncol 26: 5530-5536, 2008.

44. Gallagher DJ, Konner JA, Bell-McGuinn KM, Bhatia J, Sabbatini P, Aghajanian CA, Offit K, Barakat RR, Spriggs DR and Kauff ND: Survival in epithelial ovarian cancer: A multivariate analysis incorporating BRCA mutation status and platinum sensitivity. Ann Oncol 22: 1127-1132, 2011.

This work is licensed under a Creative Commons Attribution-NonCommercial-NoDerivatives 4.0 International (CC BY-NC-ND 4.0) License. 